\title{
"It Helps You Not Feel So Bad-Feel Like You Again": The Importance of Community for Individuals with Psychiatric Disabilities
}

\author{
Greg Townley ${ }^{1}$
}

Received: 16 February 2015/Accepted: 15 September 2015/Published online: 25 September 2015

(C) Springer India Pvt. Ltd. 2015

\begin{abstract}
Community integration refers to the notion that individuals with disabilities should have opportunities to live, work, engage with others, and enjoy recreational activities in the same manner as peers without disabilities. Community integration research has emerged as a high priority among mental health advocates, policy makers, and researchers. Currently, our understanding of community integration comes primarily from definitions and theories provided by researchers. Although these are helpful, it is important to uncover the ways in which mental health consumers understand and experience community for themselves. To this end, 30 individuals with psychiatric disabilities were engaged in semi-structured interviews about their definitions of community, the types of communities they belong to, and the relative importance they place on community inclusion and participation. It is expected that results of this study will inform our conceptualization and measurement of community integration; and call attention to the need to develop new programs or refine existing interventions to promote community integration of individuals with psychiatric disabilities.
\end{abstract}

Keywords Psychiatric disability - Community integration - Qualitative research · Community mental health $\cdot$ Recovery

Greg Townley

gtownley@pdx.edu

1 Department of Psychology, Portland State University, P.O. Box 751, Portland, OR 97207-0751, USA

\section{Introduction}

All too often, individuals with psychiatric disabilities experience isolation and lack opportunities to fulfill meaningful roles and activities in their communities [1]. This is unfortunate given strong evidence that participation in community life positively impacts health [2-4]. The concept of community integration refers quite simply to the notion that individuals with disabilities should have opportunities to live, work, engage with others, and enjoy recreational activities in the same manner as peers without disabilities [1]. Further, this focus assumes that individuals with psychiatric disabilities can live successfully as participating members in the community when they have access to supportive services that are matched to their levels of need $[1,5,6]$. Community integration research is devoted to studying individual-level and environmental/contextual factors that encourage or discourage active participation of individuals with psychiatric disabilities in community settings.

This body of research has emerged as a high priority among mental health advocates, policy makers, and researchers [7-10]. However, less is known about how individuals with lived experience of mental health challenges understand and define their community experiences, and how these experiences in turn impact their mental health recovery. This study will fill in that gap by utilizing qualitative data analysis to assess and document meanings and experiences of community for individuals with psychiatric disabilities. Study findings have implications for research and practice aimed at encouraging active participation of individuals with psychiatric disabilities in community life. 


\section{Overview of Community Integration Theory and Research}

In the twenty-first century, the ideal of individuals with disabilities enjoying equal opportunities to live and participate in their communities remains an unrealized goal. For example, despite an expanding emphasis on supporting people with psychiatric disabilities in achieving employment goals, unemployment remains the normative state. The education gap is also huge; compared to one in 10 people without disabilities who drop out of high school, one in 5 adults who have a disability do not graduate from high school [11]. This contributes to the increased likelihood that individuals with psychiatric disabilities will be forced to live below the poverty line, struggle to meet basic needs, and have little to no additional financial resources to engage in meaningful social and recreational activities in the community [12]. These barriers are further compounded by pervasive negative attitudes toward mental illness that result in discrimination in housing, employment, and social life.

In order to help address these challenges, community integration research has emerged as a high priority among a growing group of researchers studying the experiences of individuals with psychiatric disabilities living and engaging in community settings. For example, Bond and colleagues refer to community integration as "a unifying concept providing direction and vision in community mental health for people with severe mental illness" [13, p. 570]. Similarly, Yanos instructs that "first and foremost, it is important that community integration be placed on the agenda of researchers who study the effects of place on people with mental illness" [10, p. 673]; and Davidson [7, p. 243] suggests that "the relatively uncharted territory of how people with severe and persistent mental illness navigate their immediately social environments becomes both a timely and important focus for empirical study". Community integration has also been on the agenda of national mental health policy. For example, The President's New Freedom Commission on Mental Health report [11] stressed the need for services to assist persons with psychiatric disabilities to live, work, learn, and participate fully in their communities.

Community integration has traditionally been conceptualized as physical presence in the community and operationalized as the cumulative frequency of self-initiated participation in community activities and use of community resources (e.g., shopping, working, going to church, and visiting health centers) $[14,15]$. Throughout the last decade, researchers have noted that the concept of community integration should encompass more than simply being in the physical presence of the general public and participating in activities [16, 17]. Rather, it should be conceptualized as a multi-dimensional process involving physical integration (participation in activities of daily living in the broader community), social integration (social contact with community members of one's own choosing); and psychological integration (an individual's sense of community and belonging) $[1,14]$.

Researchers have also begun to discuss the degree of opportunity that people have to utilize community resources [18], assuming that individuals may not choose to access all available opportunities [19]. Rather than simply focusing on changing the beliefs and behaviors of individuals with psychiatric disabilities, this framework also includes an assessment of the types of changes that must be made to settings and systems to promote integration [18]. A related approach to conceptualizing community integration suggests that it is a process that unfolds over time in which persons increase their capacities for connectedness (e.g., enjoying reciprocal social relationships) and citizenship (e.g., enjoying the rights and responsibilities of a democratic society) [9].

Broadly, research findings indicate that community integration is linked to higher levels of well-being and quality of life, and better mental health. For example, Townley et al. [17] assessed participants' reports of involvement in community activities and quantified the geospatial spread of activities using Geographic Information Systems. They found that individuals who reported more participation in activities farther from their homes were more satisfied with their lives. The authors suggest that participating in activities in the community may provide individuals with a sense of meaning and purpose; and it likely offers them opportunities to interact with others and obtain positive social support. Townley et al. [20] found that having casual social connections with natural supports in the community (e.g., coffee shop baristas, pharmacists, and grocery store clerks) predicted mental health recovery community integration over and above the influence of more traditional types of social support (e.g., support from friends and family).

A study involving older adults with schizophrenia found that community integration was correlated with lower levels of positive symptoms and negative symptoms; and also lower levels of depression [21]. Decreased levels of psychopathology and distress were similarly noted in earlier studies [22, 23]. Community integration has also been found to be associated with less loneliness [24] and increased social relationships in the community (e.g., [20, 25]).

\section{Conceptual Framework and Research Questions}

Community integration research thus far has largely been dominated by the use of survey data, check-lists, and other 
self-report indicators to determine individuals' perceptions of their environments [26-29]. Such methods can be quite useful; and, indeed, they have helped to investigate determinants and outcomes of community integration. However, there are limits to what survey data can demonstrate. Traditional quantitative questionnaires tend to fracture experience and exclude important contextual aspects. For this reason, researches have begun to use qualitative methods (e.g., semi-structured qualitative protocols and case-studies) to capture richer, thicker descriptions of community integration (e.g., [5, 30-32]). The use of narratives from members of marginalized groups has also been recognized as an effective way to give people voice [33, 34].

The overarching goal of this study is to gain a better understanding of how individuals with psychiatric disabilities define and understand community, including the types of communities to which they claim membership and the relative importance they place on community inclusion and participation. It is expected that the results of this study will inform our conceptualization and measurement of community integration; and call attention to the need to develop new programs or refine existing interventions to promote community integration of persons with psychiatric disabilities. To achieve these goals, this study has three specific research questions.

Research Question 1: What does community mean to individuals with psychiatric disabilities?

Research Question 2: What types of communities do individuals report belonging to?

Research Question 3: Do individuals with psychiatric disabilities consider community integration to be an important component of well-being and recovery?

In examining these questions, the study intends to help uncover the meanings and significance of community integration/inclusion from the perspectives of individuals with psychiatric disabilities. Currently, our understanding of community integration comes primarily from definitions and theories provided by researchers. Although these are helpful, it is important to uncover the ways in which mental health consumers understand and experience community for themselves.

\section{Methods}

\section{Participants}

Thirty individuals were selected to participate in this qualitative study from a broader mixed-methods study assessing community integration of 100 individuals residing in supported and non-supported housing. This sample size was selected based on sampling suggestions in the qualitative literature on grounded theory (e.g., [35]). Further, the author engaged in a continuous process of determining whether theoretical saturation had been reached, meaning that sampling and data analysis continued until all concepts in the theory were well-developed and no new data appeared [35]. It was determined that theoretical saturation was reached after the initial 30 interviews were completed; thus, no additional participants were enrolled in the study.

The demographic characteristics of this sub-sample approximated the larger sample in the quantitative study (see Table 1). Participants were recruited from the following two community mental health centers in the southeastern United States: (1) the Mental Illness Recovery Center, Inc. (MIRCI), a nonprofit organization in Columbia, SC whose mission is to provide community-based services to individuals with psychiatric disabilities through mental health treatment and housing initiatives; and (2) a community mental health center affiliated with the South Carolina Department of Mental Health. To be included in this study group, individuals had to be over 18 years of age; live in supported or independent housing; have lived at their current residence for at least 1 month; and have participated in community mental health services for at least 6 months.

\section{Measures}

Qualitative research questions were assessed using a semistructured qualitative protocol. The interview probe questions were derived from the primary research questions guiding this study, which were in turn developed from a review of the relevant literature. After asking participants warm-up questions about their typical daily activities and their neighborhoods, the author engaged them in a more

Table 1 Demographics of the qualitative sub-sample compared to the full study sample

\begin{tabular}{lll}
\hline & $\begin{array}{l}\text { Qualitative } \\
\text { sub-sample }\end{array}$ & $\begin{array}{l}\text { Total } \\
\text { sample }\end{array}$ \\
\hline $\mathrm{N}$ & 30 & 100 \\
Mean age & 47.2 & 48 \\
Sex: female & $52 \%$ & $53 \%$ \\
Race: black & $48 \%$ & $57 \%$ \\
Race: white & $52 \%$ & $43 \%$ \\
Education: graduated from high school & $68 \%$ & $74 \%$ \\
Diagnosis: psychotic disorder & $47 \%$ & $45 \%$ \\
Diagnosis: mood or anxiety disorder & $53 \%$ & $55 \%$ \\
Married & $4 \%$ & $6 \%$ \\
Working & $20 \%$ & $20 \%$ \\
\hline
\end{tabular}


specific discussion about community integration, including meanings attached to the word community, perceptions of community belonging, and the importance of community integration to recovery and well-being.

Qualitative prompts were designed such that an emic understanding of community integration would emerge; that is, participants would draw from their own experiences to bring unique contextual meaning to the community integration construct. Participants were invited and encouraged to discuss other relevant topics that were not specifically covered in the prompts.

\section{Procedures}

The 30 individuals who participated in the qualitative portion of the study were randomly selected from the full study sample of 100 participants. Selected individuals were contacted via telephone and told that participation in the qualitative component of the study was optional. A full $100 \%$ of the initially selected individuals agreed to participate. Qualitative interviews were conducted at participants' residences in order to make them feel more comfortable when discussing important issues related to their housing, neighborhood, and community experiences. Further, the author's active involvement in the mental health consumer community in the city in which this study took place helped to increase participants' trust in the research process and willingness to provide open and honest information.

Each main question in the qualitative interview protocol described above was asked verbatim in order to ensure that each person discussed key topic areas that would address research questions. The probe (or follow-up) questions were intended to direct participants who either spoke very little or spoke off topic. However, the interviews were conducted more in the style of a discussion than a structured research protocol. Therefore, the interviewer followed up with other questions regarding topics discussed by participants even if those questions were not directly outlined in the interview protocol. After all questions were addressed, client participants were compensated $\$ 10$ for their participation. All interviews were audio-recorded using a digital voice recorder and transcribed verbatim by a professional transcribing company. Interviews were then entered into NVivo 10.0 qualitative coding software.

\section{Qualitative Data Preparation and Analysis}

Once the qualitative data were collected, transcribed, and entered into the NVivo software program, the coding process began. Modified grounded theory was used to code the data. Grounded theory coding involves taking only the information provided in the data to create a set of codes to describe the data without any outside influences [36]. However, given the theoretical perspective that guides the study, it was necessary for this coding work to be conducted using modified grounded theory [37, 38]. That is, theory informed but did not constrain the coding process. Through an iterative process involving transcript readings and modifications, the concepts and ideas were organized into general categories and subcategories that will be described further in the results section.

After all interviews were coded, an auditing process was employed whereby data was re-examined to look for responses that might disconfirm the codes that were created. This allowed for the refinement of existing hypotheses and consideration of alternate hypotheses in light of non-supporting or contradictory data. Analyses examined participants' descriptions of how they understand the term community, as well as their descriptions of the types of communities to which they belong. Further, analyses identified participants' experiences in the community, as well as the importance that individuals place on community integration as it relates to well-being and recovery.

\section{Reliability and Validity}

\section{Reliability}

Two major considerations are addressed here for reliability. The first is consistency in data collection and analysis methods (outlined in detail above). All participants were asked the same questions during the interview process. Further, the author conducted all of the interviews; thus, interviews were consistent in content, tone, and style. The same researcher also did all of the coding. Second, a data audit procedure was conducted for each interview and for each thematic category to assess consistency and thoroughness of coding. Specifically, coding and text excerpts were examined and those codes that had fewer text excerpts than expected were investigated to assess why particular interviews were not included. When certain codes were not used in a transcript, a negative case analysis was conducted whereby the transcript was reread to search the text for possible missed coding [37]. Disconfirming evidence (i.e., text that was not consistent with general coding patterns) was also assessed to determine whether the coding framework needed to be refined. Results of the data audit support the consistency and thoroughness of coding; and no major changes to the coding framework were necessary.

\section{Validity}

In order to ensure internal validity, the author coded all interviews using a low level of inference; that is, codes 
were assigned based on the participants' words rather than the author's preconceptions and assumptions. Further, in five cases, the author re-contacted participants to make sure his interpretation of their words was accurate. This member check allowed the author to have confidence in his understanding of the participants' words as they related to the thematic content areas.

\section{Results}

This section presents qualitative results corresponding to the primary research questions. Throughout the section, quotes from participants are provided to illustrate key thematic areas and conceptualizations of what community means to participants. Participants are identified by age, race, and gender (WF for White Females, BF for Black Females, WM for White Males, and BM for Black Males). For example, '35BM' would be used to identify a 35 yearold black male. With consent from participants, photographs are also presented to reinforce the human connection between this research and the lived experiences of study participants.

\section{Research Question 1: Definitions of community}

Merriam-Webster defines community as "the people with common interests living in a particular area"; "an interacting population of various kinds of individuals in a common location"; and "a group of people with a common characteristic or interest living together within a larger society" (2014). When asked to talk about how they define the word community for themselves, more than two-thirds of the sample (22/30) provided very similar definitions to the one provided by Merriam-Webster. For example, participants said the following when asked to define community: "I think it's where people come together with a likemindedness to help each other and themselves, you know, at the same time. It's like a sense of camaraderie; and it's also like everybody is on the same page of what they want to have happen" (56WF); and "Community means the people around you, the people you live with, the people that you socialize with" (48WM).

Three participants defined community according to the social obligations that individuals within communities have for one another. For example, one participant said, "We're a team. If there is stuff going on, we let the police know, or even with the neighbors, our own neighbors, you know, your music is too loud or something like that. I mean, we look out for one another" (42BF). Five participants' definitions of community included the specific types of communities to which they belong and from which they receive support:
Table 2 Types of Sub-communities Identified by Participants

\begin{tabular}{ll}
\hline Type of sub-community & $\begin{array}{l}\text { Number and percent of participants } \\
\text { reporting type of sub-community }\end{array}$ \\
\hline Mental health-related & $20(67 \%)$ \\
Residential & $16(53 \%)$ \\
Spiritual & $16(53 \%)$ \\
Leisure & $15(50 \%)$ \\
Substance abuse-related & $10(33 \%)$ \\
Work & $6(20 \%)$ \\
None & $2(7 \%)$ \\
\hline
\end{tabular}

$N=30$

Well a community is a group of people. To me, like the Alcoholics Anonymous fellowship is a community. The university is a community, and that happens to be my alma mater. To me, this building is a community. I mean, the people that live here, whether they're owners or residents or whatever $(65 \mathrm{WM})$.

\section{Research Question 2: Types of communities}

As noted by the participant above, individuals belong to different types of communities. Participants identified six types, or categories, of sub-communities, as summarized in Table 2. On average, participants each identified three subcommunities to which they belong, with a range of $0-7$ sub-communities (including 'none').

\section{Mental Health-Related Sub-Communities}

Mental health-related settings were the most commonly discussed sub-communities. These included psychosocial day programs, mental health treatment centers, and selfhelp organizations. Participants identified two primary types of supports provided by mental health settings. First, 20 participants $(67 \%)$ spoke of the role that mental health settings play in their recovery from mental illness.

You know, I like to tell people that MIRCI helped me because I had never been properly diagnosed. And so that's the biggest thing MIRCI did for me outside of providing me with housing when I had nowhere to go. But the other thing is with being able to see a psychiatrist regularly and having my meds monitored, it's helped me regain some humanity that I didn't have before because now I actually like helping other clients here when before I didn't want to have anything to do with anybody (39WM).

Second, participants spoke about the social support provided by mental health settings. This support included being able to see friends who also use mental health services. For example, one participant said the following: 
Well, it's people that are going through a lot of the same stuff that I am-problems with mental health or trying to get mental health services. I've got friends that go to MIRCI, and I see them on a weekly, if not daily, basis. So, it's good to see them (49BM).

Social support was also identified in the form of friendly interactions with mental health service providers in the community:

As a matter of fact, I ran into my psychiatrist at Captain D's [a restaurant], and we talked for a few minutes. It was just like a regular guy, he wasn't all formal with me. That was nice. I mean, I knew he was nice, but you think he's so doctor this and that, but he's also very likable (52BF).

Although participants reported primarily positive characteristics of mental health-related sub-communities, five participants $(17 \%)$ did discuss their frustration with certain aspects of this sub-community. Most of this frustration had to do with not feeling adequately supported by staff, having to switch medications too often, and feeling different from other clients at the mental health center:

And I meet people there [the mental health center]their mental illness is very clear, and it's evident. It's very evident. I don't know if this will come off wrong, but I don't feel that mine is evident, and to that degree. Sometimes I feel uncomfortable around them. Sometimes I really try to stay away from them (48WF).

\section{Residential Sub-Communities}

Participants' descriptions of residential sub-communities ( $\mathrm{n}=16,53 \%$ ) focused on the role of housing environments in their lives. Participants spoke about social norms in their neighborhoods, responsibilities they have to residential communities, and activities they enjoy doing near their homes. One aspect of residential sub-communities that was discussed in a positive manner by several participants was the presence of diversity at their housing sites.

For example, one participant said the following:

I think the thing I like most about it, specifically the neighborhood here, is the diversity. It's not a whitebread suburban sort of environment where, you know, all the houses look the same, all the people look the same. You know, you have a lot of diversity in terms of just cultural-you know, I can drive down Diamond Blvd. and be exposed to 30 different cultures in the course of an hour. I love that! (39WM).

Other participants, particularly those in congregate housing environments, spoke of wishing there was more diversity in their housing sites in terms of both health-status and demographic characteristics: "I would prefer to live with other people that do not have a mental illness because I feel that sometimes it makes me sicker to be around some of them. It would also be nice to have some younger people here" (31WF).

\section{Spiritual Sub-Communities}

Over half of the participants $(n=16,53 \%)$ discussed spiritual sub-communities. Churches were described as offering numerous types of support, including emotional support (e.g., helping individuals to cope with the death of loved ones); material/tangible support (e.g., providing them with food and clothing); and social support (e.g., inviting them out to dinner or to see movies in the community). One participant discussed her church as a particularly important support system when she has a mental health crisis:

Well, my main community is the church, which is not that far from here, and I have a lot of close friends there. And they take care of me when I'm sickwhen I need mental health services or do need to go in the hospital. They help get me there, bring me clothes, take care of my cat (67WF).

In contrast, other participants addressed concerns about negative attitudes from church members about their mental illnesses. This experience was particularly salient for a participant who attended a local Mormon church:

The older generation doesn't understand the autistic child, or the girl with depression, or an adult that's going through the substance abuse. They think-they believe in the older church which was if you had a substance abuse or if you had a mental illness or you had a child with autism, you are the devil. And they still-the older generation still seems to judge (31WF).

\section{Leisure/Recreational Sub-Communities}

One-half of participants discussed leisure sub-communities ranging from social media sites (e.g., Facebook and Meetup.com), recreational activity settings (e.g., being a member of the Columbia Museum of Art and attending openings and exhibits), and hobby groups (e.g., being a member of local arts groups and selling hand-made crafts). These sub-communities were discussed in a positive manner by most participants, as they offer opportunities for socializing, creative outlet, and intellectual enrichment:

... It's just a really great group of people here in the Columbia art community...You know what my 
favorite part is? You get to see and meet the artists, and you learn about the work process in their head. Is it something that's just captivating them at the moment, or is it something from their roots, their family or something? And some people are inspired by words, emotion, and flowers and things... It's just layers upon layers of things that fascinate me about it (52BF).

However, some participants discussed feeling uncomfortable at leisure activity locations due to factors such as age and socioeconomic status: "I used to love going to play bingo, but now it's all young kids who want to play for money. I barely got money for bills-definitely not for betting on bingo!" (73BM). Other participants spoke of concerns related to how the social norms of their leisure groups might negatively impact their recovery from substance abuse. For example, when one participant shares her artwork at local events, she feels out of place at times because there is often alcohol present: "It seems like everything is centered around drinks, and there's this part of me that knows it might be dangerous for me to be socializing to that extent where that drinking goes on" (48WF). Another participant shared similar concerns when he goes to see local live music:

Unfortunately, every band in the world plays in a bar, so that's the one thing I have to really watch out for is that I don't start going to places just to drink...The problem with addiction is you have to rewire your brain to say that you can go see a band without drinking (39WM).

\section{Substance Abuse-Related Sub-Communities}

One-third of participants (10/30) listed substance-abuse groups, such as Alcoholics Anonymous (AA) and Narcotics Anonymous (NA) as important sub-communities. Participants who attend AA and/or NA reported interacting with this sub-community numerous times per week, both at formal meetings and in social settings outside of meetings. Generally, AA and NA meetings were regarded as environments accepting of anyone who is committed to sobriety. However, participants did mention a slight tension between individuals in recovery from substance abuse and individuals in recovery from co-occurring disorders (i.e., both mental illness and substance abuse issues). Similarly, participants spoke of occasional disagreements at the meetings due to the diversity of people in attendance: "It's a range of ages, ethnicities, and what not. People genuinely want to help you, but we're human, and we can be cliquey and talk about each other" (48WF).

\section{Work Sub-Communities}

Given the relatively low number of study participants who were employed, work environments were the least-reported sub-community $(\mathrm{n}=6,20 \%)$. Although one participant reported work as a stressor ("I work four days a week overnight which can be really stressful, especially because I think I need my sleep more than most people do") (45WM), the other five participants who discussed work-related subcommunities spoke of work as a positive, normalizing experience that keeps them on a regular schedule:

Prior to getting a job, I got so sick and tired of being here [the home] all the time. I didn't know what the solution was, but someone suggested that I try working. It worked out, and now I just love being able to contribute to my household. And I feel like it makes me more productive in other areas of my life too $(43 \mathrm{WF})$.

Besides offering structure and routine, participants noted that working provides them with opportunities to cultivate interests and relationships that would not have otherwise been available to them. For example, one participant grew frustrated with the lack of vocational assistance offered at the psychosocial day program he attends, so he decided to seek out assistance from the Small Business Development Center (SBDC). The participant explained, "There's not a lot of people I can run ideas by that would understand where I'm coming from, but the SBDC has been great in helping me get my website advertising business up-andrunning" (43WM). He has even been asked to teach workshops related to starting small businesses as a mental health consumer.

\section{No Sub-Communities}

Although the vast majority of participants had little difficulty identifying and describing sub-community memberships, two participants had a difficult time identifying any sub-communities they belong to within the broader community. One participant viewed social groups or hobbies as niches but not necessarily as communities:

I never felt a need to think that way about a community. When you say that community is like social groups or ethnic, or whatever, I more think of clubs. But a community is more personal, you know, it's more like-I mean that's the whole thing. It's like, when I think of community, and I'm looking out this window, I just see people-not necessarily communities of mental health consumers or communities of car collectors. I just consider those cliques or 
niches-less than a community, a lot less. That stuff doesn't matter to me (45WM).

Another participant expressed feelings of not belonging anywhere: "Sometimes I feel like I don't belong anywhere to be honest... Because everywhere I go, I get talked about or picked on" (33WF). Although these experiences represent the minority of attitudes from study participants, it is important to consider alternative viewpoints, particularly as they relate to individuals' ability or desire to engage in community life.

\section{Research Question 3: Importance of Community Integration}

After discussing specific community integration experiences, interviews with participants ended with a discussion of the importance of community integration to their recovery and well-being. Out of 30 participants, only two participants $(7 \%)$ said that community integration experiences are not important to them:

I'm not driven to be a part of anything. I guess I'm too much of a loner. That's how I've grown up, and that's how I'll always be. I mean, you know, when you get this-when you get a mental illness, it's pretty serious. It kinda just makes you want to be alone (45WM).

Maybe if I had people supporting me I would want to do more, but I don't. It's not fun having depression. No matter how much medicine you can take, there's nothing you can do about it. At this point, I think it's best for me to just stay in here by myself (33WF).

The remaining 28 participants were vocal in the importance they place upon engaging in activities in the community. Twenty-two participants reported that community integration facilitates their mental recovery-alleviating symptoms and helping them feel better:

When I had my relapse, right after I got out of the hospital, I went right back to work. I realized that I didn't want to stay round my apartment because, you know, when you be in all day and all night, that's enough to lose it. Sometimes just the change of scenery makes it better (42BF).

Well, I'd feel a whole lot sicker if I had to stay inside and was afraid to go outside. That really was crucial. There was a time that I could have just died, never gotten out of bed, but then I decided it was more important for me to take classes at school, at college. And so there were literally times I'd walk, counting each step, and I'd-I can't tell you how gutwrenching that is, but that's what I had to do, and I knew I had to do it, or just give up forever (55BM).
Six participants also discussed the positive effects of community engagement on their self-esteem and goal attainment:

I think it gives me something to focus on. It gives me the opportunity to put goals out in front of methings that I can accomplish, and when I do, I gain mastery and feel good about it. So, I think, for me, I think doing this-starting this business has been a real positive thing, and it's gonna get me out more into interacting with people as well (43WM).

It helps your self-esteem and also helps you feeling like you belong to people. It helps you not feel so bad-feel like you again (43WF).

Further, interacting with others in the community can enhance worldview and ability to relate to different types of people. For example, one participant struggled for years with paranoia-thinking that people were out to harm him, and also thinking that no one was worth getting to know. He lived in his car in the woods and had little to no human interaction for almost 3 years. His reintegration into society has been life-changing in many regards, including his opinions of fellow community members:

It helps add to the sense of purpose. It helps for me to get out and see other people and realize that not everybody is worthless, not everybody is sheep, oreverybody is an individual and everybody has their own lives, and it's just, you know, interesting. And now I love just watching people, watching how they interact. So that helps-getting out and seeing others (39WM).

Another participant echoed these words and shared a touching story about her experiences trying new types of foods and dining styles:

It helps me to get along with more people, understanding and observing people. I like to see different things...I need to branch out and learn different people's customs because I find myself embarrassed sometimes. I had went out to dinner with this friend, and they had a finger bowl, and I didn't know what it was. And I felt ashamed. So, I saw him washing his hands in it, and I knew what to do. But I didn't know what to do before he washed his hands. I wondered, do I sip it? Oh well, I know now! And I like learning those things $(52 \mathrm{BF})$.

Finally, a few participants even noted the positive impact that their own community integration experiences can have on others, suggesting a sense of agency and influence that likely increases their perceptions of connection to the community: 
At Christmastime I make special things up for the homeless and take them to the shelter. And I also pray for them and try to listen when I go in. And I tell them to aim high and stay in the program because it can help them get back on their feet (52BF)

You know, Five Points has always been a very special place to me. I've seen it go through a lot of changes, and I like to think that people around here recognize me and are happy seeing a familiar face. Sometimes I'll even put a few coins in the meter if I have some in my pocket and someone is about to get a ticket $(65 \mathrm{WM})$.

\section{Discussion}

This study represents one of the first attempts to define and assess the importance of community integration from the perspectives of individuals with lived experience of mental health challenges. It also differs from previous studies by tapping into personal understandings of community integration rather than relying on definitions and conceptualizations proposed in the literature. Results highlight participant definitions of community, the types of communities individuals belong to, and the importance of integration to well-being and recovery.

\section{Summary of Major Findings}

Broadly, participants' definitions of community corresponded closely to traditional dictionary definitions, suggesting that individuals with psychiatric disabilities conceptualize the term in a similar manner as individuals in the general population. Participants discussed several types of sub-communities to which they belong within the larger community. The most commonly reported sub-communities were mental health-related (e.g., psychosocial day programs, self-help organizations, and mental health treatment centers). At first blush, this finding appears to be discouraging given that community integration research and practice aspires to have people engage in activities and settings not tied to mental health treatment. However, closer inspection of participants' descriptions of mental health-related sub-communities suggests that these settings can serve as a bridge to the broader community. For example, a few participants discussed interacting with mental health staff when they see them in community settings (e.g., grocery stores and restaurants); receiving transportation from mental health staff to activity locations; and appreciating that mental health services have helped make them healthy enough to engage with others in the community. These findings speak to the importance of assessing the role of traditional and non-traditional mental health services in facilitating opportunities for community integration and participation.

In addition to sub-communities tied to mental health services, participants noted other types of sub-communities not necessarily tied to services, including residential, spiritual, leisure, and work settings. These community memberships reflect the importance of what consumer/survivor activist Pat Capponi [39] has referred to as "a home a job, and a friend." Participants spoke about the important role their residential communities play in providing stability and support, as well as exposing them to diverse people, places, and activities. Similarly, individuals who worked spoke of employment as a positive, normalizing experience that keeps them on a regular schedule and allows them to cultivate interests and relationships. Finally, participants noted the social benefits of engaging in leisure activities, spiritual communities, and hobby groups, although certain factors such as age, socioeconomic status, and recovery-specific characteristics (e.g., maintaining sobriety) were noted as potential barriers to inclusion in some social settings. Given the range of community types to which individuals reported belonging, it is important that future research measures and examines the impact that membership in multiple types of communities has on a variety of well-being outcomes.

One of the intended contributions of this study is to provide evidence of the importance of community integration from the perspectives of individuals with lived experience of mental health challenges. This perspective is lacking in the literature, as researchers and practitioners tend to assume that community integration is important and of interest to all individuals with psychiatric disabilities. Resoundingly, participants reported that community integration is important to their well-being and recovery from mental illness. Individuals reported that engaging in activities helps to keep them from isolating in their homes and makes them feel healthy and alive. Community experiences also boost self-esteem and competence by offering individuals responsibilities and activities on which to focus time and energy. Finally, interacting with community members provides individuals with opportunities to learn about diverse groups, expand their social horizons, and influence the well-being of the community. Although these reports come from a small sample, the fact that $93 \%$ of the sample endorsed the value of community integration suggests that it is a fruitful area for continued research, practice, and policy work.

\section{Practical Implications of Findings}

Given evidence of the central role that community integration plays in the lives of individuals with psychiatric disabilities, it is important to assess rehabilitative 
approaches to promoting participation and inclusion. One suggestion is to make community integration a priority when formulating treatment plans for clients [40]. Individualized treatment plans can include client-directed goals for number of activities to perform on a weekly or monthly basis, number of job/housing applications to complete, timelines for enrolling in college classes, and so forth. Additionally, rehabilitative services tied to mental health settings may encourage individuals to integrate into the community through provision of skills that help individuals socialize with others, fulfill activities of daily living (e.g., shopping and cleaning), and obtain and maintain education and employment [8]. Besides relationships tied to mental health settings, evidence from this study and the literature suggests the importance of working with individuals to bolster networks of relationships with individuals in the broader community. These may include friends and family members [33, 41]; relationships tied to housing (e.g., neighbors and landlords) [29]; relationships at various activity settings (e.g., co-workers, congregation members, and members of social/hobby groups) [32]; and even casual community relationships developed via regular contact with other individuals who live and work in the community (i.e., distal supports) [20, 42].

\section{Limitations and Future Directions}

A few study limitations should be taken into consideration when interpreting findings. First, the cross-sectional nature of the study design limits strong conclusions about causality and trajectories for change. Given that community integration is a process that unfolds over time, it is advisable to perform longitudinal studies in which individuals are interviewed periodically as they are discharged from hospitals or overcome relapses and begin to live in independent settings, engage in community activities, and develop or re-form social relationships.

Future longitudinal studies should also further examine the processes by which individuals alter existing communities or seek out new communities to support their recovery and quality of life.

Second, the generalizability of results may be limited both because of the small sample size and the location of the research in an urban setting in the southeastern United States. To gain insight on the applicability of the findings beyond the southeastern region, this study should be replicated in other areas of the U.S. and internationally. Another potential limitation related to generalizability of results is that, given ethical considerations in the research, the study only included people who agreed and were able to be interviewed. Accordingly, the perspectives of individuals who did not wish to participate or who were not stable at the time of the interview may be missing.
Finally, this study only includes the perspectives of individuals with psychiatric disabilities. There is a lack of triangulation from other sources of information that may further illuminate the important role of community in the lives of individuals with psychiatric disabilities. Future studies should include the perspectives of diverse stakeholders, including mental health staff, family members, landlords, and other community members. For example, studies should ask mental health staff how they view and support their clients' community integration experiences. Although this has been explored some in the literature [43, 44], there is a lack of clarity regarding whether staff are more of a help or a hindrance in clients' attempts to seek out and engage in community living and activities.

\section{Conclusions}

It is important to understand community integration from the perspectives of individuals with lived experience of mental health challenges in order to make recommendations for interventions and service delivery. This study highlights definitions of community, types of communities individuals belong to, and the importance of community integration and participation to well-being and recovery. Given evidence of the importance of community integration reported in the research literature, endorsed by mental health service organizations, and reflected in the words and experiences of mental health consumers, it can be tempting to provide prescriptive, "one-size fits all" suggestions related to community integration processes. However, the following interchange between the author and a research participant underscores the importance of understanding and respecting individual differences and preferences for when, how, and with whom individuals choose to engage in community life:

Participant: Well, I'll be honest with you. I know the community is healthy, and I really enjoy it when I choose to be a part of it. It does me a world of good knowing it's out there when I need it. I'm not the social butterfly by any stretch of the imagination. I know how to communicate with people, but I do like my solitude also. I'm okay being by myself.

Author: Yeah, that makes a lot of sense. Correct me if I'm wrong, but it sounds to me like you're saying that it comes down to choice-that if you want to participate in the community, you like doing that, but it needs to be on your terms and when you feel like you want to do it.

Participant: Yeah, you got that right. That's it in a nutshell. 
Acknowledgments Research was supported by a Fahs-Beck Fund for Research and Experimentation grant from the New York Community Trust. Thanks to staff and consumers of the Mental Illness Recovery Center, Inc. and South Carolina Department of Mental Health for making this research possible.

\section{References}

1. Wong Y-L, Solomon P. Community integration of persons with psychiatric disabilities in supportive independent housing: conceptual model and methodological issues. Ment Health Serv Res. 2002;4(1):13-28.

2. Barrera M. Social support research in community psychology. In: Rappaport J, Siedman E, editors. Handbook of community psychology. New York: Kluwer Academic; 2000. p. 215-46.

3. Fothergill KE, Robertson ME, Green KM, Thorpe RJ, Juon H-S. Effects of social integration on health: a prospective study of community engagement among African American women. Soc Sci Med. 2011;72(2):291-8.

4. Strauss JS, Carpenter WT. Predictions of outcome in schizophrenia: III. Five-year outcome and its predictors. Arch Gen Psychiatry. 1977;34:159-63.

5. Yanos PT, Barrow SM, Tsemberis S. Community integration in the early phase of housing among homeless persons diagnosed with severe mental illness: success and challenges. Community Ment Health J. 2004;40(2):133-50.

6. Rog DJ. The evidence on supported housing. Psychiatr Rehabil J. 2004;27(4):334-44.

7. Davidson L. More fundamentally human than otherwise. Psychiatry. 2005;63(3):243-9.

8. Nelson G, Lord J, Ochocka J. Empowerment and mental health in community: narratives of psychiatric consumer/survivors. J Community Appl Soc Psychol. 2001;11:124-42.

9. Ware NC, Hopper K, Tugenberg T, Dickey B, Fisher D. Connectedness and citizenship: redefining social integration. Psychiatr Serv. 2007;58(4):469-74.

10. Yanos PT. Beyond "landscapes of despair": the need for new research on the urban environment, sprawl, and the community integration of persons with serious mental illness. Health Place. 2007;13:672-6.

11. United States. President's New Freedom Commission on Mental Health. Achieving the promise: transforming mental health care in America. Publication number SMA-03-3832. Rockville, MD: US Department of Health and Human Services; 2003.

12. Drake RE, Skinner JS, Bond GR, Goldman HH. Social security and mental illness: reducing disability with supported employment. Health Aff. 2009;28(3):761-70.

13. Bond FR, Salyers MP, Rollins AL, Rapp CA, Zipple AM. How evidence-based practices contribute to community integration. Community Ment Health J. 2004;40:569-88.

14. Aubry T, Myner J. Community integration and quality of life: a comparison of persons with psychiatric disability in housing programs and community residents who are neighbors. Can $\mathbf{J}$ Commun Ment Health. 1996;15(1):5-20.

15. Cummins RA, Lau ALD. Community integration or community exposure? A Review and discussion in relation to people with an intellectual disability. J Appl Res Intellect Disabil. 2003; 16:145-57.

16. Gulcur L, Tsemberis S, Stefancic A, Greenwood RM. Community integration of adults with psychiatric disabilities and histories of homelessness. Community Ment Health J. 2007;43(3):211-28.

17. Townley G, Kloos B, Wright PA. Understanding the experience of place: expanding methods to conceptualize and measure community integration of persons with serious mental illness. Health Place. 2009;15(2):520-31.

18. Salzer MS, Baron RC. Who is John? Community integration as a paradigm for transformative change in community mental health. In: Nelson G, Kloos B, Ornelas J, editors. Community psychology and community mental health: towards transformative change. New York: Oxford University Press; 2014. p. 228-37.

19. Yanos PT, Felton BJ, Tsemberis S, Frye VA. Exploring the role of housing type, neighborhood characteristics, and lifestyle factors in the community integration of formerly homeless persons diagnosed with mental illness. J Ment Health. 2007;16(6):703-17.

20. Townley G, Miller H, Kloos B. A little goes a long way: the impact of distal social support on community integration and recovery of persons with psychiatric disabilities. Am J Community Psychol. 2013;52:84-96.

21. Abdallah C, Cohen CI, Sanchez-Almira M, Reyes P, Ramirez P. Community integration and associated factors among older adults with schizophrenia. Psychiatr Serv. 2009;60(12):1642-8.

22. Silverman CJ, Segal SP. Who Belongs? An analysis of ex-mental patients' subjective involvement in the neighborhood. Adult Resid Care. 1994;8(2):103-13.

23. Timko C, Moos RH. Outcomes of the treatment climate in psychiatric and substance abuse treatment programs. J Clin Psychol. 1998;54(8):1137-50.

24. Granerud A, Severinsson E. The struggle for social integration in the community - the experiences of people with mental health problems. J Psychiatr Ment Health Nurs. 2006;12:288-93.

25. Kruzich JM. Community integration of the mentally ill in residential facilities. Am J Community Psychol. 1985;13(5):553-64.

26. Nagy MP, Fisher GA, Tessler RC. Effects of facility characteristics on the social adjustment of mentally ill residents of boardand-care homes. Hosp Community Psychiatry. 1988;39(12):1281-6.

27. Prince PN, Gerber GJ. Subjective well-being and community integration among clients of assertive community treatment. Qual Life Res. 2005;14:161-9.

28. Sherman SR, Frenkel ER, Newman EW. Community participation of mentally ill adults in foster family care. J Community Psychol. 1986;14:120-33.

29. Townley G, Kloos B. Examining the psychological sense of community for individuals with serious mental illness residing in supported housing environments. Community Ment Health J. 2011;47(4):436-46.

30. Bromley E, Gabrielian S, Brekke B, Pahwa R, Daly KA, Brekke JS, Braslow JT. Experiencing community: perspectives of individuals diagnosed as having serious mental illness. Psychiatr Serv. 2013;64(7):672-9.

31. Browne G. Housing, social support, and people with schizophrenia: a grounded theory study comparing boarding houses and private homes. Issues Ment Health Nurs. 2005;26:311-26.

32. Royce-Davis JC. "It's the day-to-day living that matters": the meaning and process of community in the lives of a couple with significant psychiatric disabilities. Am J Community Psychol. 2001;29(6):807-32.

33. Boydell KM, Gladstone BM, Crawford ES. The dialectic of friendship for people with psychiatric disabilities. Psychiatr Rehabil J. 2002;26(2):123-31.

34. Lord J, Schnarr A, Hutchison P. The voice of the people: qualitative research and the needs of consumers. Can J Commun Ment Health. 1987;6(2):25-36.

35. Creswell JW. Qualitative inquiry and research design: choosing among five approaches. Thousand Oaks, CA: Sage; 2012.

36. Strauss AL, Corbin J. Basics of qualitative research: grounded theory procedures and techniques. Newbury Park, CA: Sage; 1980. 
37. Kloos B, Gross SM, Meese KJ, Meade CS, Doughty JD, Hawkins DD, Zimmerman SO, Snow DL, Sikkema KJ. Negotiating risk: knowledge and use of HIV prevention by persons with serious mental illness living in supported housing. Am J Community Psychol. 2005;36(3-4):357-72.

38. Miles M, Huberman A. Qualitative Data Analysis. Thousand Oaks, CA: Sage; 1994.

39. Capponi P. Upstairs in the crazy house. Toronto: Harper Collins Canada; 1992.

40. Badger TA, McNiece C, Bonham E, Jacobson J, Gelenberg AJ. Health outcomes for people with serious mental illness: a case study. Perspect Psychiatr Care. 2003;39(1):23-32.
41. Freedman RI, Fesko SL. The meaning of work in the lives of people with significant disabilities: consumer and family perspectives. J Rehabil. 1996;62:49-55.

42. Wieland ME, Rosenstock J, Kelsey SF, Ganguli M, Wisniewski SR. Distal support and community living among individuals diagnosed with schizophrenia and schizoaffective disorder. Psychiatry. 2007;70(1):1-11.

43. Abraham KM, Stein $\mathrm{CH}$. Case managers' expectations about employment for people with psychiatric disabilities. Psychiatr Rehabil J. 2009;33(1):9-17.

44. Casper ES, Carloni C. Assessing the underutilization of supported employment services. Psychiatr Rehabil J. 2007;30:182-8. 